\title{
Effect of the Source of Carbon and Vitamin C Present in Tropical Fruits, on the Production of Cellulose by Gluconacetobacter Xylinus
}

\author{
Olga Perna Manrique*, Ruben Jaramillo Lanchero and Liliana Vitola Garrido \\ Facultad de Educación y Ciencias, Universidad de Sucre cra 28 \# 5-267 Sincelejo, Colombia; liliana.vitola@unisucre. \\ edu.co, olga.perna@unisucre.edu.co, ruben.jaramillo@unisucre.edu.co
}

\begin{abstract}
Objective: To evaluate the effect of Carbon source and Vitamin C present in tropical fruits, on the production of Cellulose by Gluconacetobacter xylinus. Methodology and Statistical Analysis: A Variance Analysis of three factors was carried out in the applied factorial design, to determine if three types of fruit- in their different concentration- affect the production of Cellulose in a given period of time. Where the Wet Weight is the Dependent Variable. And the Independent Variables are: first, the Days of Incubation (3, 7, 14, 21 and 28 days); second, the three fruits analyzed (Mango, Creole Cherry and Guava); and, third, the Concentrations of $20 \%$ and $40 \%$ of the extract of each fruit. Findings: After analyzing the results of content of Vitamin C and sugars for each fruit (Cherry, Guava and Mango)- and in relation to the results obtained- it was found that, contrasting these two parameters, the content of Gluconic Acid for Cherry decreased by 89.67\%; for Guava, 69.7\%; and for Mango 87.6\%. The above corroborates that the higher the content of Vitamin C and Sugars, the greater is the production of the Biopolymer (Cellulose), and the amount of Gluconic Acid decreases in the culture medium at each incubation time, maintaining the $\mathrm{pH}$ of the medium without altering its buffering function too much. Applications: With this, it will be possible to demonstrate the possibility of synthesizing Bacterial Cellulose by Gluconacetobacter xylinus, in a culture medium containing non-conventional sources of Carbon, such as the extracts of three tropical fruits such as Mango, Guava and Creole Cherry. In addition, through this study, it will be possible to compare the values of Gluconic Acid obtained from the three extracts, showing their relationship with the amount of Vitamin C present in the extracts.
\end{abstract}

Keywords: Bacterial Cellulose, Carbon Source, Culture Medium, Tropical Fruits, Vitamin C

\section{Introduction}

Cellulose is the constituent element of wood. It is a natural polymer formed by units of Glucose. Cellulose of vegetable origin represents for the paper industry the main raw material in the production of products such as cardboard, paper, fabrics, rayon and cellulose acetate. To that end, large areas of forest hectares are cut annually in the world, which weakens the soil, causes the extinction of native flora and fauna, and produces an increase in global warming, causing a direct impact on the Carbon Cycle ${ }^{1}$. Cellulose can be synthesized by different biological systems such as Agrobacterium, Achromobacter, Aerobacter, Azotobacter, Pseudomonas, Rhizobium,
Sarcina and Acetobacter, which produce Cellulose as part of their normal metabolic processes $\underline{2,3}$. Bacterial Cellulose represents the purest form of Cellulose and has unique properties ${ }^{4.5}$. In particular, its physical-chemical and mechanical characteristics are superior to those of Vegetal Cellulose ${ }^{-6}$. It is produced aerobically, in the form of film in static cultures and in the form of pellets in shaken cultures. And it is the strict aerobic gram negative bacterium Gluconacetobacterxylinus, the model organism due to its growth in different substrates ${ }^{\underline{7}}$.

Due to the multiple uses of Cellulose synthesized by Gluconacetobacter xylinus, such as food additive (thickener, emulsifier), in Biomedicine (treatment of burns,

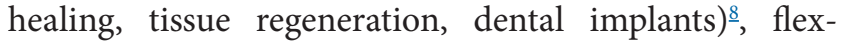

*Author for correspondence 
ible demonstration screens ${ }^{2}$, composite paper reinforced and electronic ${ }^{10}$, among others, research has been done replacing the traditional sources of Carbon (Fructose, Sucrose and Glucose) by other unconventional sources, such as Glycerol, Sorbitol, Mannitol, Lactose, Galactose, Ethanol, Maple Syrup, Molasses Caña, Beet Molasses, Fruit Juices (Pineapple, Asian Pear, Papaya, Mango, Apple, Grapes, Cashew), Ascorbic Acid, Peptone, Corn Liquor, Yeast Extract, AmorpHopHallus rivieri Durieu (Konjac Powder), Agroindustrial Waste, Sugar Makes $\underline{11-14}$ in order to generate more economical growing media and to increase production, due to the low yield obtained so far, in such a way that it can be scaled at the industrial level and can develop methods of production of Bacterial Cellulose (CB) with a lower cost.

In tropical countries, the consumption of seasonal fruits is important, but there are also pulpers that process the pulp and subject it to pasteurization to be frozen or refrigerated and sell it in supermarkets. However, the use of husks and seeds are converted into waste that is usually taken to local landfills. The importance of this Biotechnological Proposal lies in the fact that the results can make progress in the industrial exploitation of the Cellulose synthesized by G. xylinus in perspective to replace the use of Vegetable Cellulose, due to the contaminating process that means the bleaching of Cellulose and also part of the pulp of imported cellulose and of the obtained one at national level. This work is a contribution to the knowledge in the physiological and biochemical behavior of G. xylinus in the most efficient conditions of growth and production of Cellulose, positively influencing the improvement of the Environment and the Carbon Cycle.

\section{Methodology}

The experimental procedures were carried out in the Microbiology Research Laboratory, Microorganisms Biology Research Group (GIBM), Department of Biology and Chemistry, Faculty of Education and Sciences, Universidad de Sucre, Sincelejo, Colombia.

\subsection{Biological Material}

The wild strain Gluconacetobacter xylinus IFO 13693which was used in the present investigation- was donated by Dr. Edgardo Escamilla Marván (R.I.P.) of the Cellular Physiology Institute of the Universidad Autónoma de México (UNAM).

\subsection{Maintenance of the Strain of G. xylinus}

For the maintenance of the strain,repiques were made in the culture medium adapted to $\mathrm{pH}$ 5.6. In two $50 \mathrm{ml}$ flasks of sterile culture medium, to which the inoculum was added. Subsequently, they were incubated at room temperature until the cellulose film was observed. Then they were transferred to a culture medium of $200 \mathrm{ml}$, every 15 days, to obtain an axenic culture medium and use it as an inoculum in subsequent experiments.

\subsection{Bacterial Cellulose Production}

For the culture medium, author in $\frac{15}{}$ were adapted to $\mathrm{pH}$ between 4-6, changing the Sucrose by extracts of Mango, Guava and Creole Cherry. In this case, the inoculum was seeded in $200 \mathrm{ml}$ of medium, at concentrations of $20 \%$ and $40 \%$, for Mango, Creole Cherry and Guava in triplicate, for a total of 30 reactors for each of the aforementioned sources of Carbon. Then, they were sterilized in a pot-type autoclave. After this, they cooled and were taken to the sowing chamber leaving them for 15 minute under UV light. Next, the bacterium was planted from the culture medium where the strain is maintained. From this was taken $20 \mathrm{ml}$ (10\% of the inoculum) that were added in each of the bioreactors. They were left in incubation at room temperature, measuring the thickness of the cellulose film every 3, 7, 14, 21 and 28 days, in order to determine which of these concentrations has the best performance in terms of the thickness of the films.

\subsection{Wet Weight}

Once the thickness of the CB film was determined, it was again washed with distilled water to remove impurities and partially dried with absorbent paper to determine, thus, the Wet Weight, using an analytical balance.

\subsection{Dry Weight}

After determining the Wet Weight the Cellulose film was washed with $0.1 \mathrm{~N} \mathrm{NaOH}$ at $30^{\circ} \mathrm{C}$ for 20 minutes to remove or completely dissolve the cells trapped in the $\mathrm{CB}$ film. Then they were dried in a furnace $\left(220^{\circ} \mathrm{C}\right.$ laboratory oven, 28 liters jouan, model: EU28) at $60^{\circ} \mathrm{C}$, placing them on filter paper for 3-6 hours, taking care to change their position to obtain a uniform drying until constant weight. 


\subsection{Determination of Compressibility of Synthesized Cellulose}

Washed and dried films were subjected to the application of a weight of $2000 \mathrm{~g}$ for 30 seconds (modified Budhiono method $)^{16} . v=[1-p 2 / p 1] * 100$. Where $\mathrm{p} 2$ is the Wet Weight after having applied the weight of $2000 \mathrm{~g}$ and $\mathrm{p} 1$ is the Wet Weight obtained before applying the $2000 \mathrm{~g}$.

\section{$2.7 \mathrm{pH}$ Measurement}

The $\mathrm{pH}$ of the culture media was determined by taking small volumes of medium in the different incubation times during the $\mathrm{CB}$ production process, in order to determine the $\mathrm{pH}$ variations that occur during this process and thus establish the optimum conditions that the microorganism needs for its growth. A pH potentiometer (Ohaus $\mathrm{PH}$ Meter, Starter Series) is used for this measurement.

\subsection{Determination of Gluconic Acid in the Samples}

An aliquot of $2.5 \mathrm{ml}$ of the remaining culture medium and the same procedure as the standard procedure was followed, according to Table 1. Finally, this determination was made for the samples collected during the fermentation times: $3,7,14,21,28$ days.

Table 1. Preparation of the gluconic acid standard curve

\begin{tabular}{|c|c|}
\hline Absorbancia & concentración de ácido glucónico \\
\hline 0,337 & 0,0248 \\
\hline 0,422 & 0,0495 \\
\hline 0,526 & 0,0742 \\
\hline 0,613 & 0,099 \\
\hline 0,675 & 0,1238 \\
\hline 0,715 & 0,1485 \\
\hline
\end{tabular}

\subsection{Measurement of the Carbon Source from the Quantification of Glucose}

A volume of the remaining medium from each of the bioreactors was taken at the different culture times and centrifuged at 15,000 $\mathrm{rpm}$ (ENGELSDORF/LEIPZIG ${ }^{\circ}$ DDR-7123) for 30 minutes, to sediment the suspended solids, and $3 \mathrm{ml}$ of the supernatant were used in the immediate quantification of Glucose stored at $-70^{\circ} \mathrm{C}$, for later analysis. Glucose was determined by the Oxidase/ Peroxidase Glucose method, for which the AA Enzymatic Glicemia Kit, Wiener $\mathrm{Lab}^{\circledR}$, containing a standard
Glucose solution of $(100 \mathrm{mg} / \mathrm{Dl})$ was used. The principle is in the conversion of Glucose by the action of GlucoseOxidase in Gluconic Acid and Hydrogen Peroxide which, in the presence of Peroxidase, oxidizes the chromogen (4-Aminoantipyrine/phenol) in a red compound. The absorbance of the samples was measured at a wavelength of $505 \mathrm{~nm}$ and a temperature of $37^{\circ} \mathrm{C}$. This reading was made in a spectrophotometer (Thermo Scientific UV-Visible Spectrophotometer Series GENESYS ${ }^{\circledR}$ 10S)

\subsection{Statistical Analysis}

A Variance Analysis of three factors was carried out in the applied factorial design, to determine if three types of fruit in their different concentrations affect the production of Cellulose in a given period of time. Where the Wet Weight is the Dependent Variable. And the Independent Variables are: first, the Days of Incubation $(3,7,14,21$ and 28 days); second, the three fruits analyzed (Mango, Creole Cherry and Guava); and, third, the Concentrations of $20 \%$ and $40 \%$ of the extract of each fruit.

Hypothesis according to different factors, where iterations were raised and applied a level of significance of 5\%

\section{Results}

After obtaining the inocula that allowed the activation of G. xylinus, the respective inoculation of the bacteria was done in the three extracts used (Mango, Guava and Creole Cherry) to be able to evaluate the physicochemical parameters previously described in the $3,7,14,21$ and 28 days.

\subsection{Among these is the Humid Weight}

A higher concentration of fruit extract shows a higher Humid Weight, the CreoleCherry having a value of 137.96 grams (Table 2), followed by Mango, with 128.2 grams (Table 3); and Guava, with 62.1 grams (Table 4)

Table 2. Humid weight of the films in $20 \%$ and $40 \%$ Creole cherry extract

\begin{tabular}{|c|c|c|}
\hline \multirow{2}{*}{ Day } & \multicolumn{2}{|c|}{$\begin{array}{c}\text { Humid weight (gram) } \\
\text { (Creole Cherry) }\end{array}$} \\
\cline { 2 - 3 } & $20 \%$ & $40 \%$ \\
\hline 3 & 0,28 & 0 \\
\hline 7 & 10,9 & 5,92 \\
\hline 14 & 26,1 & 56,12 \\
\hline 21 & 55,3 & 97,56 \\
\hline 28 & 81,2 & 137,96 \\
\hline
\end{tabular}


Table 3. Humid weight of the films in $20 \%$ and $40 \%$ guava extract

\begin{tabular}{|c|c|c|}
\hline \multirow{2}{*}{ Day } & \multicolumn{2}{|c|}{$\begin{array}{c}\text { Humid weight (gram) } \\
\text { (Guava) }\end{array}$} \\
\cline { 2 - 3 } & $20 \%$ & $40 \%$ \\
\hline 3 & 0,652 & 1,58 \\
\hline 7 & 2,7 & 3,86 \\
\hline 14 & 6,62 & 37,8 \\
\hline 21 & 7,1 & 45,9 \\
\hline 28 & 10,2 & 62,1 \\
\hline
\end{tabular}

Table 4. Humid weight of the films in $20 \%$ and $40 \%$ mango extract

\begin{tabular}{|c|c|c|}
\hline \multirow{2}{*}{ Day } & \multicolumn{2}{|c|}{$\begin{array}{c}\text { Humid weight (gram) } \\
\text { (Mango) }\end{array}$} \\
\cline { 2 - 3 } & $20 \%$ & $40 \%$ \\
\hline 3 & 6,4 & 4,12 \\
\hline 7 & 19,8 & 17,8 \\
\hline 14 & 59 & 95,4 \\
\hline 21 & 70,5 & 114,6 \\
\hline 28 & 78,03 & 128,2 \\
\hline
\end{tabular}

\subsection{In Relation to the Dry Weight}

After drying the films, they were again weighed, where a greater Dry Weight of 13.8 grams was found for the Cellulose film with $40 \%$ Creole Cherry extract (Table 5), followed with 12.67 grams for Mango (Table 6) and 5.4 grams for Guava (Table 7).

Table 5. Dry Weight of the films in $20 \%$ and $40 \%$ in creole cherry extract

\begin{tabular}{|c|c|c|}
\hline \multirow{2}{*}{ Day } & \multicolumn{2}{|c|}{$\begin{array}{c}\text { Dry weight (gram) } \\
\text { (Creole Cherry) }\end{array}$} \\
\cline { 2 - 3 } & $20 \%$ & $40 \%$ \\
\hline 3 & 0,03 & 0 \\
\hline 7 & 1,04 & 0,48 \\
\hline 14 & 2,5 & 5,3 \\
\hline
\end{tabular}

\begin{tabular}{|c|c|c|}
\hline 21 & 6,6 & 9,6 \\
\hline 28 & 8,2 & 13,8 \\
\hline
\end{tabular}

Table 6. Dry weight of the films in $20 \%$ and $40 \%$ in guava extract

\begin{tabular}{|c|c|c|}
\hline \multirow{2}{*}{ Day } & \multicolumn{2}{|c|}{$\begin{array}{c}\text { Dry weight (gram) } \\
\text { (Guava) }\end{array}$} \\
\cline { 2 - 3 } & $20 \%$ & $40 \%$ \\
\hline 3 & 0,058 & 0,15 \\
\hline 7 & 0,243 & 0,38 \\
\hline 14 & 0,65 & 3,74 \\
\hline 21 & 0,71 & 4,1 \\
\hline 28 & 0,97 & 5,4 \\
\cline { 2 - 3 } & \multicolumn{2}{|c|}{} \\
\cline { 2 - 3 } & &
\end{tabular}

Table 7. Dry weight of the films in $20 \%$ and $40 \%$ mango extract

\begin{tabular}{|c|c|c|}
\hline \multirow{2}{*}{ Day } & \multicolumn{2}{|c|}{$\begin{array}{c}\text { Dry weight (gram) } \\
\text { (Mango) }\end{array}$} \\
\cline { 2 - 3 } & $20 \%$ & $40 \%$ \\
\hline 3 & 0,71 & 0,41 \\
\hline 7 & 1,88 & 1,72 \\
\hline 14 & 5,67 & 9,34 \\
\hline 21 & 6,91 & 11,21 \\
\hline 28 & 7,02 & 12,67 \\
\hline
\end{tabular}

\section{$3.3 \mathrm{pH}$ Measurement}

The $\mathrm{pH}$ of the extracts of Creole Cherry, Guava and Mango as a source of Carbon decreased with time (Table 8).

\subsection{Determination of Gluconic Acid in the Samples}

For the three extracts (Creole Cherry, Guava and Mango) and the two concentrations, the measurement of Gluconic Acid was made. The results obtained will be recorded in Tables 9-15, respectively.

Table 8. PH measurement of the remaining cultures in different days and extracts (mango, guava and creole cherry)

\begin{tabular}{|c|c|c|c|c|c|c|}
\hline \multirow{2}{*}{ Days } & \multicolumn{2}{|c|}{ Mango Extract } & \multicolumn{2}{c|}{ Guava Extract } & \multicolumn{2}{c|}{ Creole Cherry Extract } \\
\cline { 2 - 7 } & $20 \%$ & $40 \%$ & $20 \%$ & $40 \%$ & $20 \%$ & $40 \%$ \\
\hline 3 & 4,65 & 4,66 & 4,84 & 5,17 & 4,78 & 5,21 \\
\hline 7 & 4,46 & 4,58 & 4,53 & 4,39 & 4,46 & 5,15 \\
\hline 14 & 4,38 & 4,51 & 4,33 & 4,16 & 4,23 & 4,78 \\
\hline 21 & 4,33 & 4,32 & 4,32 & 4,16 & 4,46 & 3,8 \\
\hline 28 & 4,29 & 4,22 & 4,15 & 4,14 & & 4,46 \\
\hline
\end{tabular}


Table 9. Values for the standard gluconic acid curve

\begin{tabular}{|c|c|}
\hline Absorbance & [] Of Gluconic Acid. \\
\hline 0,337 & 0,0248 \\
\hline 0,422 & 0,0495 \\
\hline 0,526 & 0,0742 \\
\hline 0,613 & 0,099 \\
\hline 0,675 & 0,1238 \\
\hline 0,715 & 0,1485 \\
\hline
\end{tabular}

Table 10. Gluconic acid of creole cherry in a concentration of $20 \%$

\begin{tabular}{|c|c|c|}
\hline Days & $\begin{array}{c}\text { Concentration } \\
(20 \%)\end{array}$ & Absorbance \\
\hline 3 & 4,507202786 & 1,698 \\
\hline 7 & 2,696216558 & 1,126 \\
\hline 14 & 2,126325788 & 0,946 \\
\hline 21 & 1,138515118 & 0,634 \\
\hline 28 & 0,286845021 & 0,365 \\
\hline
\end{tabular}

Table 11. Gluconic acid of creole cherry in a concentration of $40 \%$

\begin{tabular}{|c|c|c|}
\hline Days & Concentration (40\%) & Absorbance \\
\hline 3 & 8,634478392 & 1,638 \\
\hline 7 & 6,361247428 & 1,279 \\
\hline 14 & 5,481082792 & 1,14 \\
\hline 21 & 1,85911034 & 0,568 \\
\hline 28 & 1,7514643 & 0,551 \\
\hline
\end{tabular}

Table 12. Gluconic acid of guava in a concentration of $20 \%$

\begin{tabular}{|c|c|c|}
\hline Days & Concentration (20\%) & Absorbance \\
\hline 3 & 1,021370904 & 0,597 \\
\hline 7 & 0,866233972 & 0,548 \\
\hline 14 & 0,536963749 & 0,444 \\
\hline 21 & 0,448314073 & 0,416 \\
\hline 28 & 0,426151654 & 0,409 \\
\hline
\end{tabular}

Table 13. Gluconic acid of guava in a concentration of $40 \%$

\begin{tabular}{|c|c|c|}
\hline Days & Concentration (20\%) & Absorbance \\
\hline 3 & 5,778692417 & 1,187 \\
\hline 7 & 3,163526991 & 0,774 \\
\hline 14 & 2,960899161 & 0,742 \\
\hline 21 & 1,859110337 & 0,568 \\
\hline 28 & 1,751464303 & 0,551 \\
\hline
\end{tabular}

Table 14. Gluconic acid of mango in a concentration of $20 \%$

\begin{tabular}{|c|c|c|}
\hline Days & Concentration (20\%) & Absorbance \\
\hline 3 & 1,787557385 & 0,839 \\
\hline 7 & 1,138515118 & 0,634 \\
\hline 14 & 0,536963749 & 0,444 \\
\hline 21 & 0,426151654 & 0,409 \\
\hline 28 & 0,365996517 & 0,39 \\
\hline
\end{tabular}

Table 15. Gluconic acid of mango in a concentration of $40 \%$

\begin{tabular}{|c|c|c|}
\hline Days & Concentration (40\%) & Absorbance \\
\hline 3 & 4,402722811 & 1,665 \\
\hline 7 & 3,405413962 & 1,35 \\
\hline 14 & 1,167009656 & 0,643 \\
\hline 21 & 0,929555169 & 0,568 \\
\hline 28 & 0,543295868 & 0,446 \\
\hline
\end{tabular}

\subsection{Measurement of the Carbon Source from the Quantification of Glucose}

The values obtained from Glucose in this experiment are recorded in Table 16. Its relevant units are $(\mathrm{mg} / \mathrm{l})$.

Table 16. Glucose quantification consumed for measurement of the carbon source

\begin{tabular}{|c|c|c|c|}
\hline Fruta & Días & $\mathbf{2 0 \%}$ & $\mathbf{4 0 \%}$ \\
\hline \multirow{4}{*}{ Cereza } & 3 & 7,5 & 3,3 \\
\cline { 2 - 4 } & 7 & 41,3 & 13,4 \\
\cline { 2 - 4 } & 14 & 42 & 43,5 \\
\cline { 2 - 4 } & 21 & 63 & 57,8 \\
\hline \multirow{4}{*}{ Guayaba } & 28 & 89 & 71,5 \\
\cline { 2 - 4 } & 3 & 1,8 & 2,2 \\
\cline { 2 - 4 } & 7 & 6,75 & 8,6 \\
\cline { 2 - 4 } & 14 & 45,4 & 19,8 \\
\hline \multirow{4}{*}{ Mango } & 21 & 51,5 & 47,6 \\
\cline { 2 - 4 } & 28 & 60,1 & 61,5 \\
\cline { 2 - 4 } & 3 & 1,25 & 3,5 \\
\hline & 14 & 12,4 & 12,8 \\
\hline & 21 & 29,8 & 37,4 \\
\hline & 28 & 51,5 & 52 \\
\hline
\end{tabular}


According to the statistical analysis, at a level of significance of 5\%, it can be affirmed that there is not enough statistical evidence in the sample to accept the null hypothesis- the day in which the experiment was applied, does not affect the type and percentage of fruit used in the experiment, which indicates that the day in which the experiment was applied does affect the type and percentage of fruit used in it. That is, there is an iteration between the day, the type and the percentage of fruit used in the experiment or, in other words, the type and percentage of fruit used is affected by the day the experiment was applied. In addition, it can be said that regardless of the type and percentage of fruit used on the 28th day of collection of the sample, the highest levels of cellulose production were recorded.

On the third and seventh day, the production of Cellulose is almost zero for the concentrations of Cherry at $20 \%$, Cherry at $40 \%$ and Guava at $20 \%$.

The lowest cellulose productions are recorded in the concentration of Guayaba at 20\%, for days 7, 14, 21 and 28.

The highest productions of Cellulose are recorded on day 28, with the concentrations of Cherry at $40 \%$ and Mango at $40 \%$.

When the concentration goes from $20 \%$ to $40 \%$, the production of Cellulose increases on days 14, 21 and 28 , for all fruits. But, when the concentration goes from $20 \%$ to $40 \%$, on days 3 and 7, the production of Cellulose decreases for Cherry and Mango; while for Guava it increases. It is also possible to propose some linear models based on these results. The following are the results of the analysis of variance, to determine the relationship between the percentage of glucose consumed, the concentration of gluconic acid, and the production of cellulose, through linear models (Figure 1).

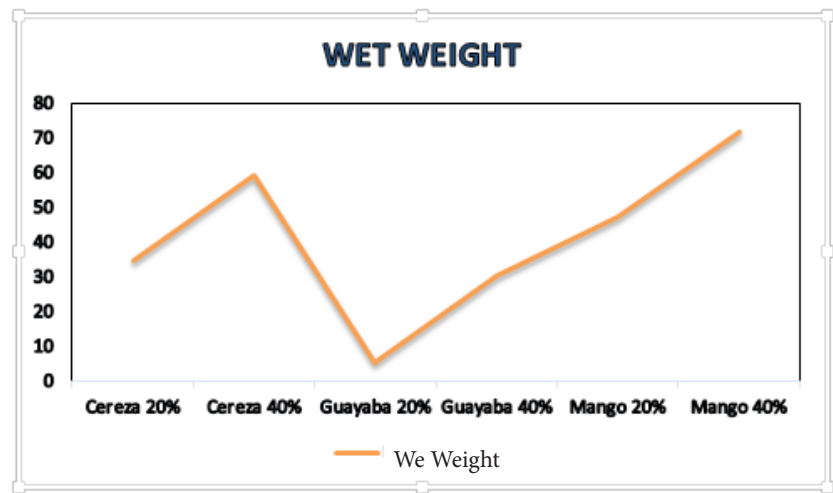

Figure 1. \% of fruit vs wet weight.
$\mathrm{Y}=:$ "Cellulose Production (Wet weight in grams)". X1 $=:$ "Consumed Glucose (\%)" X2 =: "Concentration of Gluconic Acid (g/ ml)" The following are the results the Equations (1).

$$
\begin{aligned}
& y=-5.6530+0.9341 X_{1}-2.3177 X_{2}(\text { Cherry } 20 \%) \\
& y=18.428+1.561 X_{1}-4.032 X_{2}(\text { Cherry } 40 \%) \\
& y=1.0753+0.1356 X_{1}-0.1450 X_{2}(\text { Guava } 20 \%) \\
& y=11.3874+0.8882 X_{1}-1.8456 X_{2}(\text { Guava } 40 \%) \\
& y=56.717+0.516 X_{1}-30.525 X_{2}(\text { Mango } 20 \%) \\
& y=63.062+1.246 X_{1}-15.509 X_{2}(\text { Mango } 40 \%)
\end{aligned}
$$

\section{Discussion}

According to the results obtained, it is possible to establish that the means with Creole Cherry and Mango constitute a better source of Carbon for the production of Cellulose by Gluconacetobacter xylinus, due to significant differences in content of Carbohydrates and Vitamin C. Difference is found significant in the weight obtained with the first two and the Guava because the latter contains lower percentage of Sugars. This weight corresponded between $8 \%$ and $10 \%$ of the Wet Weight of the Cellulose films, similar to previous studies where the constant Dry Weight is around 10\%. This could have resulted because the bacterium Gluconacetobacter xylinus is able to convert Carbohydrates into Acetic Acid for respiratory metabolism involving the oxidation of Ethanol to Acetic Acid and the conversion of Glucose to Gluconic Acid. However, it remains in the optimal $\mathrm{pH}$ range for the production of Bacterial Cellulose, which is between 4 and $6^{17}$. In relation to the $\mathrm{pH}$ results obtained, a clear relationship with the content of Gluconic Acid present in the medium is shown of culture, product of the metabolism of the sugars that are part of it, and that the bacteria uses to form the microfibrils that make up the biopolymer. One drawback of this culture method is the ability of Acetobacter cells to convert Glucose into Gluconic and Ketogluconic Acids, so the Glucose of the medium is consumed to the detriment of the yield for Cellulose and leads to $\mathrm{pH}$ levels that affect cell viability $\underline{18}$.

After analyzing the content results of Vitamin $\mathrm{C}$ and Sugars for each fruit (Cherry, Guava and Mango), and in relation to the results obtained, it was found that, contrasting these two parameters, the content of Gluconic 
Acid for Cherry decreased in $89.67 \%$; for Guava, by 69.7\%; and for Mango, by $87.6 \%$. The above corroborates that, the higher the content of Vitamin C and Sugars, the greater the production of the Biopolymer (Cellulose), and decreases the amount of Gluconic Acid in the culture medium in each incubation time, maintaining the $\mathrm{pH}$ of the medium without altering too much its buffer function. These results are similar to those obtained who added Ascorbic Acid (Vitamin C) to the HestrimSchramm medium, calling it HAS medium, where the content of Gluconic Acid decreased and the $\mathrm{pH}$ remained in relation to the results where only Hestrim-Schramm (HS) medium is used, without the addition of Ascorbic Acid. A marked decrease in $\mathrm{pH}$ and an increase in the content of Gluconic Acid was observed, thus showing the importance and the novelty of this study, since the Vitamin $\mathrm{C}$ used is the one that naturally contains each fruit, without the addition of artificial Vitamin C, giving an added value to the remnants of unattractive fruits for human consumption, in local markets. The ability of the cells of G. xylinus to convert the available glucose in the culture media in Gluconic and Ketogluconic Acids is known, which means that the glucose is removed from the medium to the detriment of the production of Cellulose. Based on the results obtained in this study (Table 16) it is corroborated that the Mango extract turns out to be an optimal source of Carbon for the production of Cellulose due to its high sugar content, in contrast to the sugar content present in the two other extracts (Guava and Creole Cherry). Without leaving aside that the content of Vitamin C is also important, showing the best results after performing the statistical analysis, which show that the three samples are significantly different, which vary according to each of the treatments proposed in this investigation.These coefficients indicate Equation 1 that, in the absence of gluconic acid and glucose, it is expected to obtain a cellulose production of $-5.6530 \mathrm{~g}$, that is, a cellulose loss of $5.6530 \mathrm{~g}$ would be obtained.That for each percentage point of glucose consumed, a cellulose production of $0.9341 \mathrm{~g}$ is expected.That for each $\mathrm{g} / \mathrm{ml}$ of gluconic acid, a cellulose production of -2.3177 $\mathrm{g}$ is expected, that is, a cellulose loss of $2.3177 \mathrm{~g}$ would be obtained to $20 \%$ creole cherry. That for each percentage point of glucose consumed, a cellulose production of $1,561 \mathrm{~g}$ is expected.That for each $\mathrm{g} / \mathrm{ml}$ of gluconic acid, a cellulose production of $-4,032 \mathrm{~g}$ is expected, that is, a cellulose loss of 4,032 $\mathrm{g}$ would be obtained. A model that would relate then, the amount of cellulose produced, due to the percentage of glucose and the concentration of gluconic acid present, with the cherry at $40 \%$.

This correlation shows that the relationship between the independent variable, the percentage of glucose consumed and the dependent variable, amount of cellulose produced, is direct, that is, the greater the quantity of glucose consumed, the greater the amount of cellulose produced, Guava at $20 \%$.

These coefficients indicate that, in the absence of gluconic acid and glucose, it is expected to obtain a cellulose production of $11.3874 \mathrm{~g}$. That for each percentage point of glucose consumed, a cellulose production of $0.8882 \mathrm{~g}$ is expected.That for each $\mathrm{g} / \mathrm{ml}$ of gluconic acid, a cellulose production of - $1.8456 \mathrm{~g}$ is expected, that is, a cellulose loss of $1.8456 \mathrm{~g}$ would be obtained. A model that would relate then, the amount of cellulose produced, due to the percentage of glucose and the concentration of gluconic acid present, with guava at $40 \%$.

These coefficients indicate that, in the absence of gluconic acid and glucose, it is expected to obtain a cellulose production of $56,717 \mathrm{~g}$. That for each percentage point of glucose consumed, a cellulose production of $0.516 \mathrm{~g}$ is expected.

That for each $\mathrm{g} / \mathrm{ml}$ of gluconic acid, a cellulose production of $-30,525 \mathrm{~g}$ is expected, that is, a cellulose loss of $30,525 \mathrm{~g}$ would be obtained.A model that would then relate the amount of cellulose produced, due to the percentage of glucose and the concentration of gluconic acid present, with the mango at $20 \%$.

These coefficients indicate that, in the absence of gluconic acid and glucose, it is expected to obtain a cellulose production of 63,062 g. That for each percentage point of glucose consumed, a cellulose production of $1,246 \mathrm{~g}$ is expected.That for each $\mathrm{g} / \mathrm{ml}$ of gluconic acid, a cellulose production of $-15,509 \mathrm{~g}$ is expected, that is, a cellulose loss of $15,509 \mathrm{~g}$ would be obtained.

A model that would then relate the amount of cellulose produced, due to the percentage of glucose and the concentration of gluconic acid present, with the mango $40 \%$.

All these results confirm the correlations between the participating variables.

\section{Conclusions}

It is possible to synthesize Bacterial Cellulose from Gluconacetobacter xylinus in a culture medium contain- 
ing non-conventional sources of Carbon, such as extracts of Mango, Guava and Creole Cherry, showing a higher consumption of glucose media enriched with Cherry extract. In addition, the values of Gluconic Acid obtained from the three extracts follow a behavior pattern similar to those of the standard curve. Also, they show a marked decrease with time, in relation to the amount of Vitamin $\mathrm{C}$ present in the extracts. Therefore, the higher the content of Vitamin C and Sugars present in the different media, the lower the content of Gluconic Acid. That is, they have an inversely proportional behavior, which is evidenced by the results obtained.

\section{References}

1. Brown Jr RM. Position paper microbial cellulose: A new resource for wood, paper, textiles, food and specialty products. University of Texas; 1995.

2. Tanskul S, Amornthatree K, Jaturonlak N. A new cellulose-producing bacterium, Rhodococcus sp. MI 2: Screening and optimization of culture conditions. Carbohydrate Polymers. 2013; 92(1):421-8. Crossref. PMid:23218315

3. Ullah H, Badshah M, Mäkilä E, Salonen J, Shahbazi M, Santos H. Fabrication, characterization and evaluation of bacterial cellulose-based capsule shells for oral drug delivery. Cellulose. 2017; 24(3):1445-54. Crossref.

4. Shi Q, Li Y, Sun J, Zhang H, Chen L, Chen B, et al. The osteogenesis of bacterial cellulose scaffold loaded with bone morphogenetic protein-2. Biomaterials. 2012; 33(28):66449. Crossref. PMid:22727467

5. Tyagi N, Suresh S. Production of cellulose from sugarcane molasses using Gluconacetobacter intermedius SNT-1: Optimization and characterization. Journal of Cleaner Production. 2016; 112:71-80. Crossref.

6. Ul-Islam M, Khan T, Park J. Nanoreinforced bacterial cellulose-montmorillonite composites for biomedical applications. Carbohydrate Polymers. 2012; 89(4):1189-97. Crossref. PMid:24750931
7. Nguyen V, Flanagan B, Gidley M, Dykes G. Characterization of cellulose production by a gluconacetobacter xylinus strain from kombucha. Current Microbiology. 2008; 57(5):449-53. Crossref. PMid:18704575

8. Czaja W, Krystynowicz A, Bielecki S, Brownjr R. Microbial cellulose- the natural power to heal wounds. Biomaterials. 2006; 27(2):145-51. Crossref. PMid:16099034

9. Nakagaito A, Nogi M, Yano H. Displays from transparent films of natural nanofibers. MRS Bulletin. 2010; 35(03):214-8. Crossref.

10. Jonas R, Farah L. Production and application of microbial cellulose. Polymer Degradation and Stability. 1998; 59(1-3):101-6. Crossref.

11. El-Saied H, El-Diwany AI, Basta AH, Atwa NA, El-Ghwas DE. Production And Characterization of Economical Bacterial Cellulose. Bioresources. 2008; 3(4):1196-1217.

12. Jung H, Lee O, Jeong J, Jeon Y, Park K, Kim H. Production and characterization of cellulose by acetobacter $\mathrm{sp}$ V6 using a cost-effective molasses- corn steep liquor medium. Applied Biochemistry and Biotechnology. 2009; 162(2):486-97. Crossref. PMid:19730823

13. Keshk S. Vitamin C enhances bacterial cellulose production in Gluconacetobacter xylinus. Carbohydrate Polymers. 2014; 99:98-100. Crossref. PMid:24274484

14. Ashori A, Sheykhnazari S, Tabarsa T, Shakeri A, Golalipour M. Bacterial cellulose/silica nanocomposites: Preparation and characterization. Carbohydrate Polymers. 2012; 90(1):413-8. Crossref. PMid:24751060

15. Watanabe K, Yamanaka S. Effects of oxygen tension in the gaseous phase on production and physical properties of bacterial cellulose formed under static culture conditions. Bioscience, Biotechnology, and Biochemistry. 1995; 59(1):65-8. Crossref.

16. Budhiono A, Rosidi B, Taher H, Iguchi M. Kinetic aspects of bacterial cellulose formation in nata-de-coco culture system. Carbohydrate Polymers. 1999; 40(2):137-43. Crossref.

17. Chawla PR, Bajaj IB, Survase SA, Singhal RS. Microbial cellulose: Fermentative production and applications. Food Technology and Biotechnology. 2009; 47(2):107-24.

18. Pineda LC, Mesa LC, Riascos CM. Fermentation tecniques and applications of bacterial cellulose: A review. Ingeniería y Ciencia. 2012; 8(16):307-35. Crossref. 